\title{
Sex Reversal in Reptiles: Reproductive Oddity or Powerful Driver of Evolutionary Change?
}

\author{
Clare E. Holleley ${ }^{a, b}$ Stephen D. Sarre ${ }^{b}$ Denis O'Meally ${ }^{b, c}$ Arthur Georges ${ }^{b}$ \\ ${ }^{a}$ Australian National Wildlife Collection, National Research Collections Australia, CSIRO, and ${ }^{\mathrm{b}}$ Institute for Applied \\ Ecology, University of Canberra, Canberra, A.C.T., and ${ }^{C}$ Centre for Animal Health Innovation, University of the \\ Sunshine Coast, Sippy Downs, QId., Australia
}

\section{Key Words}

Gene-environment interactions · Reptiles · Sex

determination $\cdot$ Sex reversal

\begin{abstract}
Is sex a product of genes, the environment, or both? In this review, we describe the diversity of sex-determining mechanisms in reptiles, with a focus on systems that display geneenvironment interactions. We summarise the field and laboratory-based evidence for the occurrence of environmental sex reversal in reptiles and ask whether this is a widespread evolutionary mechanism affecting the evolution of sex chromosomes and speciation in vertebrates. Sex determination systems exist across a continuum of genetic and environmental influences, blurring the lines between what was once considered a strict dichotomy between genetic sex determination and temperature-dependent sex determination. Across this spectrum, we identify the potential for sex reversal in species with clearly differentiated heteromorphic sex chromosomes (Pogona vitticeps, Bassiana duperreyi, Eremias multiocellata, Gekko japonicus), weakly differentiated homomorphic sex chromosomes (Niveoscincus ocellatus), and species with only a weak heritable predisposition for sex (Emys orbicularis, Trachemys scripta). We argue that sex reversal is
\end{abstract}

widespread in reptiles (Testudines, Lacertidae, Agamidae, Scincidae, Gekkonidae) and has the potential to have an impact on individual fitness, resulting in reproductively, morphologically, and behaviourally unique phenotypes. Sex reversal is likely to be a powerful evolutionary force responsible for generating and maintaining lability and diversity in reptile sex-determining modes.

(c) 2016 S. Karger AG, Basel

Broad commonality exists across vertebrates in gonadal structure [Morrish and Sinclair, 2002; Rhen and Schroeder, 2010], so it is not surprising that the genes and regulatory processes governing differentiation of the gonads also share a high degree of commonality across vertebrates [Sarre et al., 2004; Cutting et al., 2013]. Not so for the genes and regulatory processes that govern sex determination directing the zygote down either a male or female developmental trajectory. Variation in the mechanisms of sex determination across vertebrate species is truly remarkable, and reptiles contribute greatly to this diversity [Bachtrog et al., 2014]. Reptile sex thus presents a fertile field for investigation, providing new insights into vertebrate sex determination more generally [Gamble et al., 2015; Holleley et al., 2015; Pennell et al., 2015;

\section{KARGER}

E-Mail karger@karger.com

www.karger.com/sxd
(C) 2016 S. Karger AG, Basel

1661-5425/16/0106-0279\$39.50/0
Clare E. Holleley

Australian National Wildlife Collection

National Research Collections Australia, CSIRO

Canberra, ACT 2601 (Australia)

E-Mail clare.holleley@csiro.au 
Deakin et al., 2016; Montiel et al., 2016; Rovatsos et al., 2016].

Many reptiles have a genetic sex determination (GSD) system. Among them, many turtles and lizards have male or female heterogamety ( $\mathrm{ZW}$ and XY, XXY in some lizards), and snakes have female heterogamety (ZW, ZZW, or ZWW) [Solari, 1993; Olmo, 2005; Ezaz et al., 2010; Georges et al., 2010] (fig. 1). Reptiles also provide the only amniote examples of obligate parthenogenesis, which typically originate from interspecific hybridization [Neaves and Baumann, 2011] and some remarkable examples of facultative parthenogenesis [Booth et al., 2012]. Many other reptile species with GSD have homomorphic sex chromosomes which are difficult to differentiate cytologically [Ganesh and Raman, 1995; Ezaz et al., 2010]. Others have temperature-dependent sex determination (TSD) where sex is determined by the temperature of egg incubation with little or no evidence of a direct genetic influence [Bull, 1980; Ewert et al., 1994; Harlow, 2004]. Recently, these 2 apparently discrete modes of sex determination (GSD and TSD) have been shown to interact in some species, causing reversal of chromosomal sex [Quinn et al., 2007; Radder et al., 2008; Holleley et al., 2015]. Here, we review the occurrence of sex reversal in reptiles, its prevalence in the wild, its likely impact on our understanding of sex determination more generally, and its evolution.

\section{What Is Sex Reversal? Environmental versus Mutational Mechanisms}

Sex reversal occurs when the developmental trajectory leading to one sex under the normal genetic influence of sex chromosomes is redirected by environment or a mutation to the alternate sex. Such disruption of the regulatory processes governing the gonadal phenotype can lead to a viable, albeit alternate, sexual phenotype or to gonadal dysgenesis and compromised fitness. We restrict our review to sex reversal that leads to a viable sexual phenotype either under laboratory conditions or in the wild. Sex-reversed individuals are said to be discordant, because their phenotypic sex is the opposite of their genotypic sex. Discordance can occur in either direction: an individual can be genetically male but anatomically and functionally female or genetically female but anatomically and functionally male.

The key to unambiguously detecting sex reversal is a well-characterised sex chromosome system and genomic information on the sequence and gene content of the sex chromosomes. Widespread occurrence of homomorphic sex chromosomes in reptiles has potentially obscured instances of sex reversal in this group. For many years, the ability to adequately differentiate sex chromosomes was restricted to relatively crude G- and C-banding, or relying on differences in size or gross morphology. In cases where sex chromosomes are microchromosomes or the differences are morphologically subtle, sex chromosomes are likely to be cryptic and to go unnoticed [Ezaz et al., 2009, 2010]. More sensitive cytogenetic techniques, such as comparative genomic hybridization, have revealed formerly cryptic sex chromosomes in a wide range of species [Traut et al., 1999], including sex microchromosomes and recently evolved sex chromosomes with little morphological differentiation [Traut et al., 1999; Ezaz et al., 2005; Kawai et al., 2007]. The use of sensitive molecular cytogenetics is important because the high proportion of repetitive sequences on the heteromorphic element ( $\mathrm{Y}$ or $\mathrm{W})$ renders them particularly difficult to sequence and assemble with molecular approaches [Tomaszkiewicz et al., 2016].

One avenue of sex reversal is through mutation in the genes responsible for sex determination (e.g., master sex determining gene $S R Y$ in mammals, $D M R T 1$ or its paralogues in many other metazoans) or their associated regulatory regions. However, it is important to appreciate that sex reversal can result from changes in the expression, transmission, or reception of regulatory genes and their products well downstream of any master sex-determining gene, provided this disruption does not compromise a functional sexual phenotype. A good example of this is aromatase (CYP19A1) which is unlikely to be the master sex-determining gene in reptiles, but which, when inhibited, causes sex reversal from female to a functional male phenotype [Wibbels and Crews, 1994; Belaid et al., 2001; Britt et al., 2001; Lance, 2009]. Hence, a mutation that compromises aromatase function will potentially reverse sex without compromising the individual's viability.

The vast majority of sex chromosome research has focused upon endothermic vertebrates, the mammals and birds. These species have relatively stable sex chromosomes that are strongly differentiated, and the extrinsic environment has little or no influence over sex determination. This has led to a somewhat biased view that sex reversal occurs only as a genetic aberration through mutational processes [Eggers et al., 2014; Ohnesorg et al., 2014]. However, sex reversal can occur through both mutations and environmental influence over genes in the sex determination and/or sex differentiation cascade, the latter being the focus of this review. Emerging theory and 
increasing empirical evidence suggests that environmentally induced sex reversal is a common and naturally occurring phenomenon that can markedly and rapidly affect the evolution of species, particularly in fish [Chan and Yeung, 1983; Shao et al., 2014; McNair Senior et al., 2015] and almost certainly in amphibians [Dournon et al., 1990; Miura, 1994; Alho et al., 2010]. Sex reversal is increasingly implicated as a mechanism involved with sex chromosome lability in ectotherms [Ogata et al., 2003; Perrin, 2009; Holleley et al., 2015]. Understanding the dynamics of sex reversal is critical to understanding reptile evolution and predicting future impacts of altered climate regimes on reptilian diversity.

\section{Definitive Cases of Environmental Sex Reversal in Wild Reptile Populations}

Hormonal manipulation and exposure to exogenous chemicals can cause sex reversal in amphibians with sex chromosomes [Dournon et al., 1990; Orton and Tyler, 2015], and similar manipulations have been conducted with TSD reptiles [Bull et al., 1988; Belaid et al., 2001; Warner and Shine, 2008; Mizoguchi and Valenzuela, 2016] reversing sex from that expected given the temperature of incubation. However, the relevance of these studies to what happens in the wild remains the subject of some conjecture.

Very limited information is available on naturally occurring gene-environment interactions that affect sex determination in reptiles. Naturally occurring sex reversal in reptiles with known sex chromosomes has been observed in just 2 evolutionarily independent examples of oviparous agamid and scincid lizards.

The most extensively characterised example of reptilian sex reversal is in the central bearded dragon (Pogona vitticeps). This species has identifiable ZZ/ZW micro-sex chromosomes [Ezaz et al., 2005] and exhibits equal offspring sex ratios over most temperatures [Quinn et al., 2007; Holleley et al., 2015]. However at high incubation temperatures $\left(>32^{\circ} \mathrm{C}\right.$ ) the signal from the ZZ (nominally male) sex chromosomes is overridden, resulting in increasingly female biased sex ratios [Quinn et al., 2007; Holleley et al., 2015]. Molecular and cytogenetic techniques have definitively shown that this female bias is a result of sex reversal (feminised ZZ individuals) and is not explained by sex-specific offspring mortality [Quinn et al., 2007; Holleley et al., 2015]. Most importantly, sexreversed $P$. vitticeps occur naturally in wild populations and are reproductively viable [Holleley et al., 2015].

Sex Reversal in Reptiles
In a second case, the Eastern three-lined skink (Bassiana duperreyi), environmental conditions and chromosomal sex determination also interact to determine sex [Shine et al., 2002; Radder et al., 2008; Quinn et al., 2009]. $B$. duperreyi has a male heterogametic system of sex determination, where the Y chromosome is largely heterochromatic and significantly smaller than the X chromosome [Donellan, 1985; Matsubara et al., 2016]. Chromosomally female (XX) individuals are masculinised at low egg incubation temperatures in the laboratory [Radder et al., 2008; Quinn et al., 2009]. Ongoing research has identified high rates of sex reversal (28\% XX male) in nests of an alpine population of $B$. duperreyi (fig. 2), but it is not yet known if sex-reversed individuals of this species are reproductively viable.

These 2 cases show that sex reversal can occur in reptiles with differentiated sex chromosomes in nature. Nevertheless, these examples represent only a tiny portion of reptile species with sex chromosomes, so the prevalence of sex reversal at present cannot be properly estimated. We know of no additional published instances where rigorous temperature incubation experiments have been conducted on reptile species with well-defined sex chromosomes and where no temperature effect has been observed. Lack of published data on the effect of temperature on offspring sex ratios of GSD species may be because such experiments are not generally reported, a negative result being too obvious for publication or a positive result considered an anomaly when the experiments have been run for another purpose. We believe that this bias of incubation experimentation towards species without sex chromosomes arises in part from an underlying view that TSD and GSD are dichotomous rather than interactive states [Sarre et al., 2004].

\section{Mixed-Model Sex Determination and Inferred Sex Reversal}

While the $P$. vitticeps and $B$. duperreyi examples discussed above are the most definitive and well-studied reptile systems with sex reversal, there is a growing body of evidence that mixed-model systems, where genes and the environment interact to determine sex, are common. Sex reversal is yet to be definitively identified in these species, however, experimental and ecological evidence suggest that sex reversal is indeed occurring.

Some of the earliest evidence for the presence of mixedmodel or transitional systems of sex determination comes from the turtle Emys orbicularis. Despite this species hav- 
ing a seemingly temperature-dependent mode of sex determination [Zaborski et al., 1982, 1988], a genetic effect is detectable at the pivotal temperature for sex determination, where the influence of temperature is equivocal [ $\mathrm{Za}-$ borski et al., 1982, 1988; Dournon et al., 1990; Girondot et al., 1994]. At the pivotal temperature, the sexual phenotype is concordant with the expression of $\mathrm{H}-\mathrm{Y}$ antigens. Specifically, males are $\mathrm{H}-\mathrm{Y}$ negative $\left(\mathrm{H}-\mathrm{Y}^{-}\right)$and females are $\mathrm{H}-\mathrm{Y}$ positive $\left(\mathrm{H}-\mathrm{Y}^{+}\right)$in somatic and gonadal tissue. At other temperatures, H-Y antigens are discordant in somatic tissue, i.e., phenotypic sex and $\mathrm{H}-\mathrm{Y}$ antigen expression are decoupled presumably because temperature overrides a genetic predisposition. Thus, it appears that sex in E. orbicularis with TSD has an underlying genetic component that is overridden by temperature at extremes. Surprisingly, when field-caught animals were examined, there was a low incidence of discordance ( $6 \%$ of phenotypic females and $11 \%$ of phenotypic males) [Girondot et al., 1994]. The thermal regime in natural nests appears to favour genetic over thermal influences while also suggesting that sex reversal occurs in E. orbicularis in the laboratory and at low levels in the wild. The development of sex-linked molecular markers would be required to identify sex reversal conclusively.

In a more recently described example, the snow skink Niveoscincus ocellatus, a small live-bearing lizard occurring along a 1,200 m elevational and climatic gradient from sea level to highland regions in Tasmania, shows the hallmarks of genetic sex determination overridden by temperature [Wapstra et al., 2004, 2009; Pen et al., 2010]. Although the system of sex determination is currently unknown, populations from different elevations have different responses to maternal gestational temperature [Pen et al., 2010]. High elevation populations exhibit equal offspring sex ratios regardless of gestational temperature, whereas low elevation populations exhibit male-biased sex ratios at cool incubation temperatures. While heteromorphic sex chromosomes have not been identified in snow skinks, related species have XY heterogamety, and genetic control of sex determination is ancestral within the lineage [Donellan, 1985]. The application of comparative genomic hybridization or other high resolution cytogenetic techniques may reveal the presence of cryptic sex chromosomes and a basis for demonstrating sex reversal in this species.

In a third case, the viviparous lizard Eremias multiocellata exhibits offspring sex ratios with a female bias at low temperatures $\left(15.4 \%\right.$ male at $\left.25^{\circ} \mathrm{C}\right)$ and a male bias at high temperatures $\left(83 \%\right.$ male at $\left.35^{\circ} \mathrm{C}\right)$ [Zhang et al., 2010]. This has been interpreted as TSD, but sex chromo- somes have now been identified in this species using comparative genomic hybridization [Wang et al., 2015], suggesting that an underlying sex chromosomal system is being overridden by temperature in the laboratory. Sex-reversed individuals are yet to be identified in this species, but these data suggest that sex reversal is likely.

The evidence for sex reversal in N. ocellatus and $E$. multiocellata draws our attention to the fact that thermal effects on sex determination appear not to be limited to egg-bearing species and that sex determination in viviparous species can also be affected by the maternal gestational environment in ectotherms.

Oviparous geckos show remarkable evolutionary lability in sex chromosomes with up to 25 independent transitions having occurred [Gamble et al., 2015]. Despite clear evidence for frequent sex chromosome turnover and a now well-developed molecular tool kit to investigate sex reversal, a definitive example of sex reversal in geckos is yet to be identified [Gamble et al., 2015]. Early studies suggest the co-occurrence of heteromorphic sex chromosomes and TSD in Gekko japonicus [Yoshida and Itoh, 1974; Tokunaga, 1985]. However, these observations require verification via concurrent incubation experiments and karyotypic analysis to remove any doubt that the 2 independent studies were inadvertently conducted on different cryptic species or interspecies hybrids which are common in the area [Toda et al., 2001, 2006; Sarre et al., 2004; Gamble, 2010].

\section{The Consequences of Sex Reversal on Fitness}

Research on sex reversal has focused predominantly on mammals or birds, where reversal arises from a mutational influence on gene expression. This taxonomic bias has led to the pervasive attitude that sex-reversed reptiles are inherently defective and thus less fit [Pokorna and Kratochvil, 2016]. Inferior fitness is a reasonable assumption where reversal and subsequent breeding yields WW or YY individuals. However, in both well-documented cases of reptile sex reversal (P. vitticeps and B. duperreyi), sex reversal occurs exclusively in the homogametic sex (ZZ or XX), eliminating the potential fitness deficit associated with inviable WW or YY offspring [Bull, 1980, 1981; Quinn et al., 2011; Schwanz et al., 2013].

Experimental work in fish suggests that some fitnessrelated traits may be impaired by environmental sex reversal (growth rate) but other traits are not impaired (survivorship) [McNair Senior et al., 2012]. However, we suggest that the observed negative impact on growth rate is 
more likely to be a temporary response to the endocrine disrupting chemicals that are used to experimentally induce sex reversal [McNair Senior et al., 2012]. While comparable reptile studies are few, preliminary data suggest that sex reversal is not as detrimental as previously assumed. Indeed, ZZ sex-reversed female bearded dragons produce almost twice as many eggs as ZW concordant females [Holleley et al., 2015]. This suggests that fitness benefits flow from sex reversal.

We also know that sex reversal can have significant effects upon behavioural traits that can affect fitness [Gatewood et al., 2006; Kopsida et al., 2013; Li et al., 2016; Saunders et al., 2016]. For example, sex-reversed bearded dragons display a unique constellation of male-like and female-like morphological and behavioural traits, allowing them to respond differentially to selective pressures [Li et al., 2016]. Most importantly, sex-reversed bearded dragons are significantly bolder than both genetically concordant male and female individuals when tested for neophobia and territory exploration [Li et al., 2016]. Boldness is an important behavioural phenotype associated with fitness, because it can affect an individual's ability to find and secure mating opportunities [Mazué et al., 2015; Sih et al., 2015]. However, increased boldness carries with it a fitness trade-off in predator-rich environments, because being conspicuous may also increase the risk of mortality via predation [Sih et al., 2015].

Whether the high fecundity and increased boldness of sex-reversed dragons observed in laboratory studies translates to fitness increases in the field remains to be tested, but clearly inferior fitness of sex-reversed individuals cannot be assumed in reptiles or other vertebrates [Saunders et al., 2014, 2016]. Indeed, the reverse might be true. Characterising the life-time fitness of naturally occurring sex-reversed individuals under field conditions is the next critical step in understanding the evolutionary dynamics of this process in reptiles.

\section{Sex Reversal Maintains Lability and Diversity in Sex-Determining Modes}

The recent discovery of sex reversal in wild reptile populations has demonstrated how environmental sex reversal can precipitate the rapid evolution of temperature-dependent sex [Holleley et al., 2015]. This finding runs counter to the prevailing view that sex chromosome evolution is a unidirectional 'trap'. Under this hypothesis, TSD is the ancestral state where a species may remain or alternatively transition to a GSD mode following the acquisition of a genetic sex-determining locus. Inhibition of recombination via inversions and resultant sex chromosome differentiation then prohibits a return to the TSD state [Bull and Charnov, 1977; Bull, 1983; Pokorna and Kratochvil, 2009; Bachtrog et al., 2014]. Whilst this 'trap' model of sex chromosome evolution is compelling for mammals, in other vertebrates sex determination appears to be far more labile and changeable. Indeed, acquiring a genetic sex determining locus does not necessarily seal the fate for the chromosome on which it is borne [Wright et al., 2016]. There are multiple possible pathways for the evolution of novel sex determination modes [Quinn et al., 2011; Sarre et al., 2011; Schwanz et al., 2013]. Empirical [Holleley et al., 2015] and theoretical [Quinn et al., 2011] work suggests that all possible modes of sex determination (XY, ZW, TSD) can be easily acquired through changes in thresholds in gene expression of male- or female-determining factors alone. In an uncertain and changeable environment, labile sex-determining mechanisms may be an advantage and the process of sex chromosome turnover can result in increased diversity in the genes involved with sex determination. This process may explain why a master sex-determining gene is yet to be identified in reptiles. The repeated and evolutionarily independent capture of sex-determining loci makes it unlikely that a single gene will be implicated in sex determination across reptile clades or perhaps even between very closely related species (fig. 1). Recapitulating this point, we observe that the 3 currently identified reptile candidate sex-determining genes are different in squamates (NR5A1 P. vitticeps; Deakin et al. [2016]) and testudines (WT1 Glyptemys insculpta; Montiel et al. [2016] and CIRBP Chelydra serpentina; Schroeder et al. [2016]).

\section{Reptile Sex Reversal and Climate Change: Is the Future a World Without Males?}

There has been significant concern in the literature about the future of TSD reptiles under climate scenarios with rapid warming events [Cotton and Wedekind, 2009; Sinervo et al., 2010; Boyle et al., 2014]. This is a legitimate concern and indeed the recent discovery of an increasing rate of sex reversal in wild bearded dragon populations suggests that climatic extremes have the potential to perturb even seemingly stable GSD systems [Holleley et al., 2015]. Thus, sex ratio bias has the potential to be an extinction driver in a broader array of reptiles than first anticipated. 


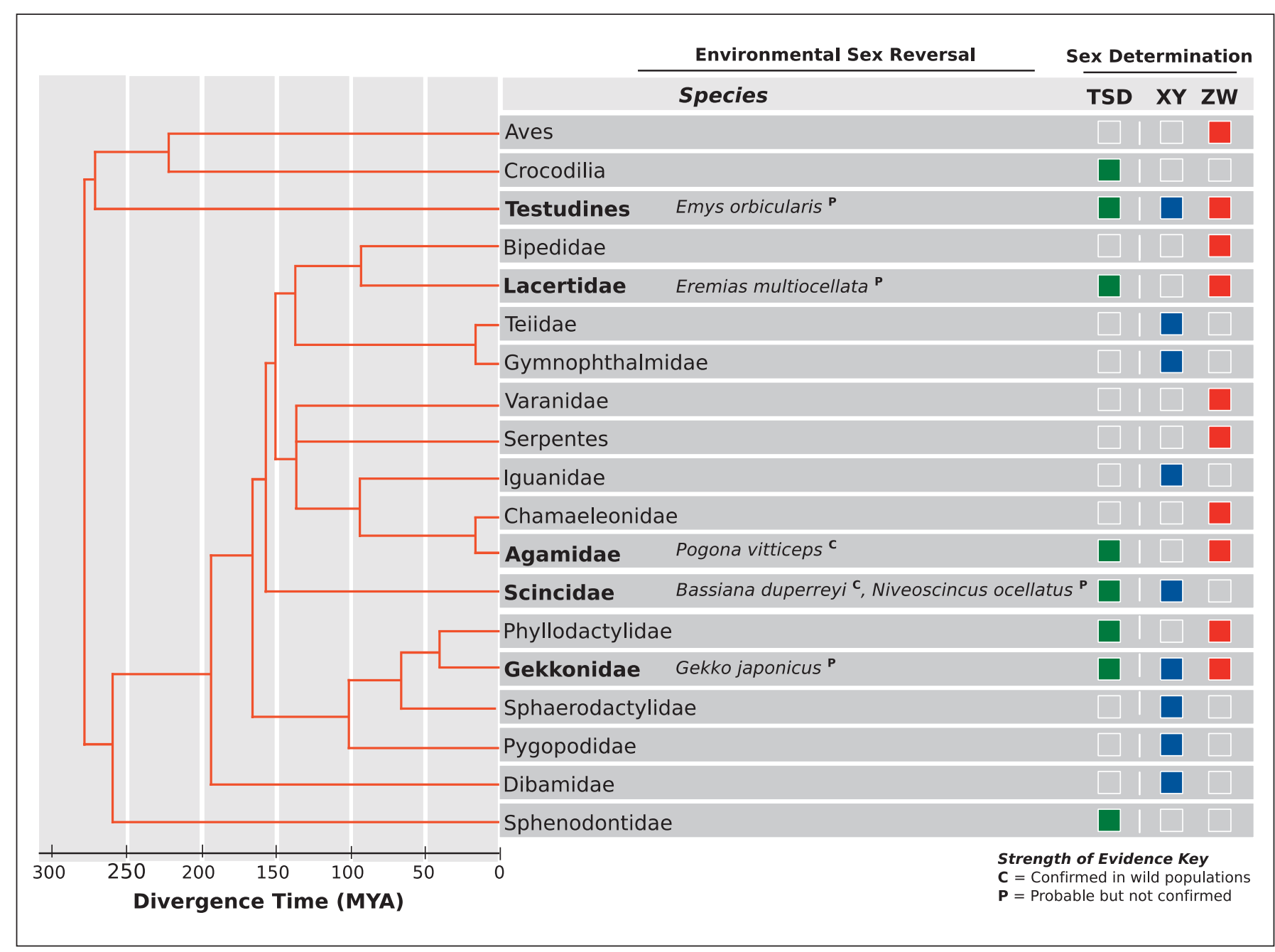

Fig. 1. The phylogenetic distribution of sex reversal in reptiles. Reptile clades are indicated in bold that have either confirmed cases of sex reversal in wild populations $\left({ }^{C}\right)$ or are known to exhibit a mixed-model mode of sex determination where sex reversal is likely $\left({ }^{\mathrm{P}}\right)$. Phylogeny adapted from Ezaz et al. [2010] and Gamble [2010]; divergence times are as per Vidal and Hedges [2009].

If global temperatures increase, will populations of reptiles become increasingly sex-biased and be susceptible to extinction by populations becoming unisexual? The answer depends on 3 things: 1) the capacity of reptiles to modify where and when they nest, 2) the capacity for thermal tolerances to adapt, and 3) the rapidity with which environmental conditions change. As temperatures rise and reptile populations experience a lower frequency of one sex (usually males), selection strongly favours individuals that can produce the rarer sex [Boyle et al., 2014]. Thus, if heritable variation in the thermal sex determination threshold exists in populations, evolution of the threshold is an effective response, reducing the risk of extreme sex ratio bias. There is significant evidence to suggest that such variation exists in TSD reptile populations, detected by estimating broad-sense heritability, narrow-sense heritability, and/or genotype-environment interactions at temperatures that produce mixed sex ratios [Bull et al., 1982; Janzen, 1992; Rhen and Lang, 1998; Janes and Wayne, 2006; McGaugh and Janzen, 2011; McGaugh et al., 2011; Rhen et al., 2011]. Supporting these heritability studies are organ culture experiments, where pairs of gonads from embryos cultured in isolation at the pivotal temperature concur in sexual phenotype, suggesting a predisposition for one sex or the other when cultured in isolation [Mork et al., 2014]. 


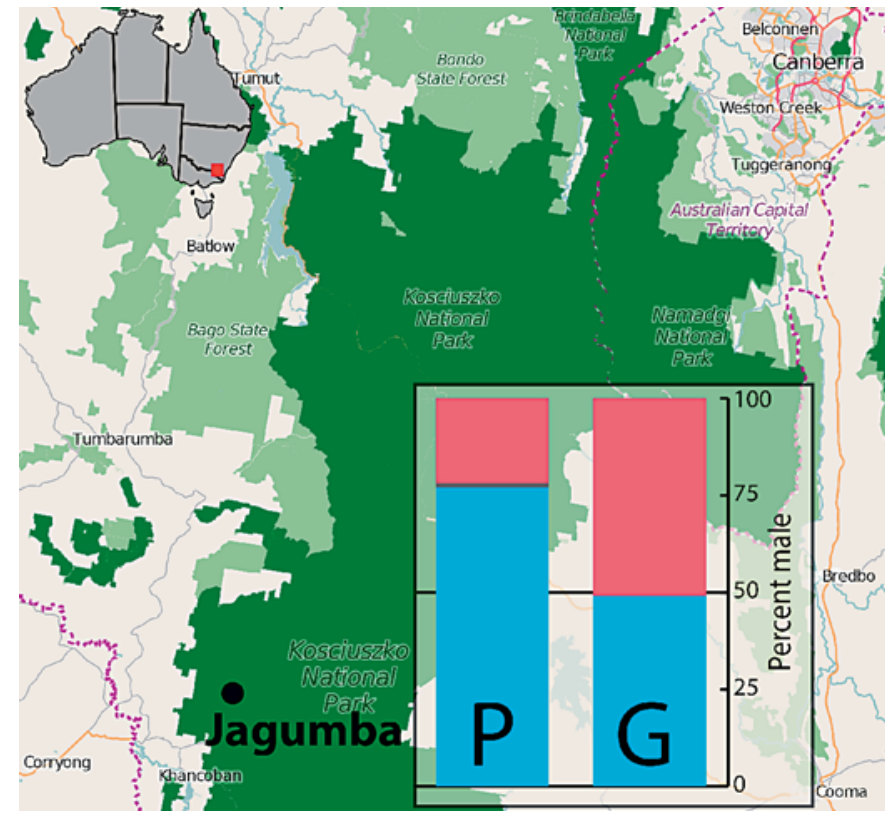

Fig. 2. Sex reversal occurs in natural alpine nests of the XY skink Bassiana duperreyi at Jagumba (1,100 m above sea level) in Kosciuszko National Park, Australia (inset). In February 2013, we opportunistically collected 114 eggs from a communal nest immediately prior to hatching. We recorded phenotypic $(\mathrm{P})$ sex by hemipene eversion [Harlow, 1996] and determined that 88 hatchlings were male (blue), 25 were female (pink), and 1 was undetermined (grey). We tested for sex reversal using PCR as described by Quinn et al. [2009] and found that the genotypic (G) sex ratio of hatchlings was $49 \%$ male, indicating that sex reversal is prevalent in this population (28\%). Specifically, we observed 25 XX females (22\%), 56 XY males (49\%), 32 sex-reversed XX males (28\%), and a single individual of indeterminate sex (1\%).

While there are alternate explanations involving maternal effects, such as intergenerational passage of sex hormones through the yolk prior to organ culture, these experiments suggest a sex-specific genetic predisposition at the pivotal temperature, where temperature is equivocal in its effect. Lastly, in P. vitticeps, a low frequency of female individuals $(<4.2 \%)$ exhibit a higher than average thermal threshold for sex reversal, producing ZZ offspring that are not feminised at high incubation temperatures [Holleley et al., 2015]. Therefore, reptile species undoubtedly have some capacity to adapt thermal sex determination thresholds. However, evolution of thermal tolerances requires successful reproduction in many successive generations. If the pace of environmental change is extremely rapid, then the risk of extreme sex ratio biases and local/species extinction may still be considerable.

\section{Conclusion}

Sex reversal occurs, or has the potential to occur, in 5 major clades of reptiles, in both XY and ZW systems, and in oviparous and viviparous species. While our knowledge of this phenomenon in reptiles is still at an early stage, it is clear that environmental sex reversal is a generalised biological process and may play an important role in reptile evolution. Indeed, it is very likely that sex reversal and gene-environment interactions are responsible for the extreme lability observed in extant reptile sex determination modes. The examples discussed here are undoubtedly the tip of the iceberg, and more cases of sex reversal will be revealed as we continue to characterise the genomic content of reptile sex chromosomes and conduct further incubation studies on reptiles with known sex chromosomes. We argue that the apparent scarcity of clear examples of reptile sex reversal is unlikely to reflect a truly low incidence of this phenomenon. Almost certainly, the low incidence is because of the scarcity of sexlinked markers and confirmed sex chromosome sequences among reptiles. The most interesting work that now needs to be done is characterising how sex reversal interacts with fundamental evolutionary processes, such as the birth and death of sex chromosomes, speciation events, and population viability under climate change.

\section{Acknowledgement}

This work was supported in part by Australian Research Council Discovery Grant DP110104377 led by A.G. We thank Xiuwen Zhang for molecular assistance generating data for figure 2 .

\section{Disclosure Statement}

The authors have no conflicts of interest to declare.

References JS, Matsuba C, Merila J: Sex reversal and pri-
mary sex ratios in the common frog (Rana
temporaria). Mol Ecol 19:1763-1773 (2010).
Bachtrog D, Mank JE, Peichel CL, Kirkpatrick M,
Otto SP, et al: Sex determination: why so
many ways of doing it? PLoS Biol 12:e1001899
(2014).
Belaid B, Richard-Mercier N, Pieau C, Dorizzi M:
Sex reversal and aromatase in the European
pond turtle: treatment with letrozole after the
thermosensitive period for sex determina-
tion. J Exp Zool 290:490-497 (2001).


Booth W, Smith CF, Eskridge PH, Hoss SK, Mendelson JR 3rd, Schuett GW: Facultative parthenogenesis discovered in wild vertebrates. Biol Lett 8:983-985 (2012).

Boyle M, Hone J, Schwanz LE, Georges A: Under what conditions do climate-driven sex ratios enhance versus diminish population persistence? Ecol Evol 4:4522-4533 (2014).

Britt KL, Drummond AE, Dyson M, Wreford NG, Jones ME, et al: The ovarian phenotype of the aromatase knockout (ArKO) mouse. J Steroid Biochem Mol Biol 79:181-185 (2001).

Bull JJ: Sex determination in reptiles. Q Rev Biol 55:3-21 (1980).

Bull JJ: Evolution of environmental sex determination from genotypic sex determination. Heredity 47:173-184 (1981).

Bull JJ: Evolution of Sex Determining Mechanisms, (Benjamin/Cummings Publishing Company, Menlo Park 1983).

Bull JJ, Charnov EL: Changes in the heterogametic mechanism of sex determination. Heredity (Edinb) 39:1-14 (1977).

- Bull JJ, Vogt RC, Bulmer MG: Heritability of sexratio in turtles with environmental sex determination. Evolution 36:333-341 (1982).

- Bull JJ, Gutzke WH, Crews D: Sex reversal by estradiol in three reptilian orders. Gen Comp Endocrinol 70:425-428 (1988).

Chan ST, Yeung WS: Sex control and sex reversal in fish under natural conditions. Fish Physiol 9:171-222 (1983).

-Cotton S, Wedekind C: Population consequences of environmental sex reversal. Conserv Biol 23:196-206 (2009).

-Cutting A, Chue J, Smith CA: Just how conserved is vertebrate sex determination? Dev Dyn 242: 380-387 (2013).

- Deakin JE, Edwards MJ, Patel H, O’Meally D, Lian $\mathrm{J}$, et al: Anchoring genome sequence to chromosomes of the central bearded dragon (Pogona vitticeps) enables reconstruction of ancestral squamate macrochromosomes and identifies sequence content of the $\mathrm{Z}$ chromosome. BMC Genomics 17:447 (2016).

Donellan SC: The Evolution of Sex Chromosomes in Scincid Lizards, Ph.D. Thesis, (Macquarie University, NSW, Australia 1985).

Dournon C, Houillon C, Pieau C: Temperature sex-reversal in amphibians and reptiles. Int $\mathrm{J}$ Dev Biol 34:81-92 (1990).

Eggers S, Ohnesorg T, Sinclair A: Genetic regulation of mammalian gonad development. Nat Rev Endocrinol 10:673-683 (2014).

-Ewert MA, Jackson DR, Nelson CE: Patterns of temperature-dependent sex determination in turtles. J Exp Zool 270:3-15 (1994).

-Ezaz T, Quinn A, Miura I, Sarre S, Georges A, Marshall Graves J: The dragon lizard Pogona vitticeps has ZZ/ZW micro-sex chromosomes. Chromosome Res 13:763-776 (2005).

Ezaz T, Moritz B, Waters P, Graves JA, Georges A, Sarre SD: The ZW sex microchromosomes of an Australian dragon lizard share no homology with those of other reptiles or birds. Chromosome Res 17:965-973 (2009).
Ezaz T, Sarre S, O’Meally D, Marshall Graves J, Georges A: Sex chromosome evolution in lizards: independent origins and rapid transitions. Cytogenet Genome Res 127:249-260 (2010).

Gamble T: A review of sex determining mechanisms in geckos (Gekkota: Squamata). Sex Dev 4:88-103 (2010).

Gamble T, Coryell J, Ezaz T, Lynch J, Scantlebury DP, Zarkower D: Restriction site-associated DNA sequencing (RAD-seq) reveals an extraordinary number of transitions among gecko sex-determining systems. Mol Biol Evol 32:1296-1309 (2015)

- Ganesh S, Raman R: Sex reversal by testosterone and not by estradiol or temperature in Calotes versicolor, the lizard lacking sex chromosomes. J Exp Zool 271:139-144 (1995).

Gatewood JD, Wills A, Shetty S, Xu J, Arnold AP, et al: Sex chromosome complement and gonadal sex influence aggressive and parental behaviors in mice. J Neurosci 26:2335-2342 (2006).

Georges A, Ezaz T, Quinn AE, Sarre SD: Are reptiles predisposed to temperature-dependent sex determination? Sex Dev 4:7-15 (2010).

Girondot M, Zaborski P, Servan J, Pieau C: Genetic contribution to sex determination in turtles with environmental sex determination. Genet Res 63:117-127 (1994).

Harlow P: A harmless technique for sexing hatchling lizards. Herpetol Rev 27:71-72 (1996).

Harlow P: Temperature-dependent sex determination in lizards, in Valenzuela $\mathrm{N}$, Lance $\mathrm{V}$ (eds): Temperature-Dependent Sex Determination in Vertebrates, pp 11-20 (Smithsonian Institution, Washington DC 2004).

- Holleley CE, O'Meally D, Sarre SD, Marshall Graves JA, Ezaz T, et al: Sex reversal triggers the rapid transition from genetic to temperature-dependent sex. Nature 523:79-82 (2015).

Janes DE, Wayne ML: Evidence for a genotype $\mathrm{x}$ environment interaction in sex-determining response to incubation temperature in the leopard gecko, Eublepharis macularius. Herpetologica 62:56-62 (2006).

Janzen FJ: Heritable variation for sex-ratio under environmental sex determination in the common snapping turtle (Chelydra serpentina). Genetics 131:155-161 (1992).

Kawai A, Nishida-Umehara C, Ishijima J, Tsuda Y, Ota H, Matsuda Y: Different origins of bird and reptile sex chromosomes inferred from comparative mapping of chicken Z-linked genes. Cytogenet Genome Res 117:92-102 (2007).

Kopsida E, Lynn PM, Humby T, Wilkinson LS, Davies W: Dissociable effects of Sry and sex chromosome complement on activity, feeding and anxiety-related behaviours in mice. PLoS ONE 8:e73699 (2013).

Lance VA: Is regulation of aromatase expression in reptiles the key to understanding temperature-dependent sex determination? J Exp Zool Part A Ecol Genet Physiol 311A:314-322 (2009).
Li H, Holleley CE, Elphick M, Georges A, Shine $\mathrm{R}$ : The behavioural consequences of sex reversal in dragons. P R Soc B 283:20160217 (2016).

- Matsubara K, O’Meally D, Azad B, Georges A, Sarre SD, et al: Amplification of microsatellite repeat motifs is associated with the evolutionary differentiation and heterochromatinization of sex chromosomes in Sauropsida. Chromosoma 125:111-123 (2016).

Mazué GP, Dechaume-Moncharmont FX, Godin JG: Boldness-exploration behavioral syndrome: interfamily variability and repeatability of personality traits in the young of the convict cichlid (Amatitlania siquia). Behav Ecol 26:900-908 (2015).

McGaugh SE, Janzen FJ: Effective heritability of targets of sex-ratio selection under environmental sex determination. J Evol Biol 24:784794 (2011).

McGaugh SE, Bowden RM, Kuo CH, Janzen FJ: Field-measured heritability of the threshold for sex determination in a turtle with temperature-dependent sex determination. Evol Ecol Res 13:75-90 (2011).

McNair Senior A, Nat Lim J, Nakagawa S: The fitness consequences of environmental sex reversal in fish: a quantitative review. Biol Rev Camb Philos Soc 87:900-911 (2012).

- McNair Senior A, Lokman PM, Closs GP, Nakagawa S: Ecological and evolutionary applications for environmental sex reversal of fish. Q Rev Biol 90:23-44 (2015).

Miura I: Sex chromosome differentiation in the Japanese brown frog, Rana japonica. I. Sexrelated heteromorphism of the distribution pattern of constitutive heterochromatin in chromosome No. 4 of the Wakuya population. Zool Sci 11:797-806 (1994).

Mizoguchi BA, Valenzuela N: Ecotoxicological perspectives of sex determination. Sex Dev 10: 45-57 (2016)

Montiel EE, Badenhorst D, Tamplin J, Burke RL, Valenzuela N: Discovery of the youngest sex chromosomes reveals first case of convergent co-option of ancestral autosomes in turtles. Chromosoma [Epub ahead of print] (2016).

Mork L, Czerwinski M, Capel B: Predetermination of sexual fate in a turtle with temperature-dependent sex determination. Dev Biol 386:264-271 (2014).

Morrish BC, Sinclair AH: Vertebrate sex determination: many means to an end. Reproduction 124:447-457 (2002).

Neaves WB, Baumann P: Unisexual reproduction among vertebrates. Trends Genet 27:81-88 (2011).

- Ogata M, Ohtani H, Igarashi T, Hasegawa Y, Ichikawa Y, Miura I: Change of the heterogametic sex from male to female in the frog. Genetics 164:613-620 (2003).

-Ohnesorg T, Vilain E, Sinclair AH: The genetics of disorders of sex development in humans. Sex Dev 8:262-272 (2014).

Olmo E: Rate of chromosome changes and speciation in reptiles. Genetica 125:185-203 (2005). 
- Orton F, Tyler CR: Do hormone-modulating chemicals impact on reproduction and development of wild amphibians? Biol Rev 90: 1100-1117 (2015).

-Pen I, Uller T, Feldmeyer B, Harts A, While GM, Wapstra E: Climate-driven population divergence in sex-determining systems. Nature 468:436-438 (2010).

-Pennell MW, Kirkpatrick M, Otto SP, Vamosi JC, Peichel CL, et al: Y Fuse? Sex chromosome fusions in fishes and reptiles. PLoS Genet 11:e1005237 (2015).

Perrin N: Sex reversal: a fountain of youth for sex chromosomes? Evolution 63:3043-3049 (2009).

$\checkmark$ Pokorna MJ, Kratochvil L: Phylogeny of sex-determining mechanisms in squamate reptiles: are sex chromosomes an evolutionary trap? Zool J Linn Soc 156:168-183 (2009).

Pokorna MJ, Kratochvil L: What was the ancestral sex-determining mechanism in amniote vertebrates? Biol Rev Camb Philos Soc 91:1-12 (2016).

$\checkmark$ Quinn AE, Georges A, Sarre SD, Guarino F, Ezaz T, Graves JA: Temperature sex reversal implies sex gene dosage in a reptile. Science 316 : 411 (2007).

Quinn AE, Radder RS, Sarre SD, Georges A, Ezaz $\mathrm{T}$, Shine R: Isolation and development of a molecular sex marker for Bassiana duperreyi a lizard with XX/XY sex chromosomes and temperature-induced sex reversal. Mol Genet Genomics 281:665-672 (2009).

-Quinn AE, Sarre SD, Ezaz T, Graves JA, Georges A: Evolutionary transitions between mechanisms of sex determination in vertebrates. Biol Lett 7:443-448 (2011).

Radder RS, Quinn AE, Georges A, Sarre SD, Shine R: Genetic evidence for co-occurrence of chromosomal and thermal sex-determining systems in a lizard. Biol Lett 4:176-178 (2008).

Rhen T, Lang JW: Among-family variation for environmental sex determination in reptiles. Evolution 52:1514-1520 (1998).

Rhen T, Schroeder A: Molecular mechanisms of sex determination in reptiles. Sex Dev 4:1628 (2010).

Rhen T, Schroeder A, Sakata JT, Huang V, Crews $D$ : Segregating variation for temperature-dependent sex determination in a lizard. Heredity 106:649-660 (2011).

Rovatsos M, Vukić J, Kratochvíl L: Mammalian X homolog acts as sex chromosome in lacertid lizards. Heredity 117:8-13 (2016).

-Sarre SD, Georges A, Quinn A: The ends of a continuum: genetic and temperature-dependent sex determination in reptiles. Bioessays 26 : 639-645 (2004).
Sarre SD, Ezaz T, Georges A: Transitions between sex-determining systems in reptiles and amphibians. Annu Rev Genom Hum Genet 12: 391-406 (2011).

Saunders PA, Perez J, Rahmoun M, Ronce O, Crochet PA, Veyrunes F: XY females do better than the XX in the African pygmy mouse, Mus minutoides. Evolution 68:2119-2127 (2014).

Saunders PA, Franco T, Sottas C, Maurice T, Ganem G, Veyrunes F: Masculinised behaviour of XY females in a mammal with naturally occuring sex reversal. Sci Rep 6:22881 (2016).

Schroeder AL, Metzger KJ, Miller A, Rhen T: A novel candidate gene for temperature-dependent sex determination in the common snapping turtle. Genetics 203:557-571 (2016).

-Schwanz LE, Ezaz T, Gruber B, Georges A: Novel evolutionary pathways of sex-determining mechanisms. J Evol Biol 26:2544-2557 (2013).

-Shao C, Li Q, Chen S, Zhang P, Lian J, et al: Epigenetic modification and inheritance in sexual reversal of fish. Genome Res 24:604-615 (2014).

-Shine R, Elphick MJ, Donnellan S: Co-occurrence of multiple, supposedly incompatible modes of sex determination in a lizard population. Ecol Lett 5:486-489 (2002).

- Sih A, Mathot KJ, Moirón M, Montiglio PO, Wolf M, Dingemanse NJ: Animal personality and state-behaviour feedbacks: a review and guide for empiricists. Trends Ecol Evol 30:50-60 (2015).

Sinervo B, Mendez-de-la-Cruz F, Miles DB, Heulin B, Bastiaans E, et al: Erosion of lizard diversity by climate change and altered thermal niches. Science 328:894-899 (2010).

Solari AJ: Sex Chromosomes and Sex Determination in Vertebrates (CRC Press, Boca Raton 1993).

Toda M, Hikida T, Ota H: Discovery of sympatric cryptic species within Gekko hokouensis (Gekkonidae: Squamata) from the Okinawa Islands, Japan, by use of allozyme data. Zool Scr 30:1-11 (2001).

Toda M, Okada S, Hikida T, Ota H: Extensive natural hybridization between two geckos, Gekko tawaensis and Gekko japonicus (Reptilia: Squamata), throughout their broad sympatric area. Biochem Genet 44:1-17 (2006).

Tokunaga S: Temperature-dependent sex determination in Gekko japonicus (Gekkonidae, Reptilia). Dev Growth Differ 27:117-120 (1985).
Tomaszkiewicz M, Rangavittal S, Cechova M, Sanchez RC, Fescemyer HW, et al: A timeand cost-effective strategy to sequence mammalian Y chromosomes: an application to the de novo assembly of gorilla Y. Genome Res 26:530-540 (2016).

- Traut W, Sahara K, Otto TD, Marec F: Molecular differentiation of sex chromosomes probed by comparative genomic hybridization. Chromosoma 108:173-180 (1999).

Vidal N, Hedges SB: The molecular evolutionary tree of lizards, snakes, and amphisbaenians. C R Biol 332:129-139 (2009).

-Wang C, Tang X, Xin Y, Yue F, Yan X, et al: Identification of sex chromosomes by means of comparative genomic hybridization in a lizard, Eremias multiocellata. Zool Sci 32:151156 (2015).

-Wapstra E, Olsson M, Shine R, Edwards A, Swain $\mathrm{R}$, Joss JM: Maternal basking behaviour determines offspring sex in a viviparous reptile. Proc Biol Sci 271 Suppl 4:S230-S232 (2004).

-Wapstra E, Uller T, Sinn DL, Olsson M, Mazurek $\mathrm{K}$, et al: Climate effects on offspring sex ratio in a viviparous lizard. J Anim Ecol 78:84-90 (2009).

Warner DA, Shine R: The adaptive significance of temperature-dependent sex determination in a reptile. Nature 451:566-568 (2008)

Wibbels T, Crews D: Putative aromatase inhibitor induces male sex determination in a female unisexual lizard and in a turtle with temperature-dependent sex determination. J Endocrinol 141:295-299 (1994).

-Wright AE, Dean R, Zimmer F, Mank JE: How to make a sex chromosome. Nat Commun 7: 12087 (2016)

Yoshida M, Itoh M: Karyotype of the gecko, Gekko japonicus. Chrom Inf Serv 17:29-31 (1974).

Zaborski P, Dorizzi M, Pieau C: H-Y antigen expression in temperature sex-reversed turtles (Emys orbicularis). Differentiation 22:73-78 (1982).

Zaborski P, Dorizzi M, Pieau C: Temperature-dependent gonadal differentiation in the turtle Emys orbicularis: concordance between sexual phenotype and serological H-Y antigen expression at threshold temperature. Differentiation 38:17-20 (1988).

Zhang DJ, Tang XL, Yue F, Chen Z, Li RD, Chen Q: Effect of gestation temperature on sexual and morphological phenotypes of offspring in a viviparous lizard, Eremias multiocellata. J Therm Biol 35:129-133 (2010). 